
\title{
R Reserach S Suare \\ Prospective data rule against osteotomy for preventing retear in patients post-ACL reconstruction
}

\author{
K. Donald Shelbourne \\ Rodney W. Benner \\ Jonathan A. Jones \\ Tinker Gray
}

\section{Video Abstract}

Keywords: The American Journal of Sports Medicine, anterior cruciate ligament, ACL, patellar tendon autograft, PTG, posterior tibial slope, PTS, graft tear, contralateral ACL tear, radiography, medial tibial plateau, posterior tibia, osteotomy, ACL injury, ACL reconstruction, revision surgery, graft failure, primary surgery, reinjury, retear

Posted Date: October 13th, 2021

DOl: https://doi.org/10.21203/rs.3.rs-966142/v1

License: (c) (i) This work is licensed under a Creative Commons Attribution 4.0 International License. Read Full License 


\section{Abstract}

For many patients undergoing reconstruction of the $\mathrm{ACL}$, the path toward recovery can be rough. One complication patients face is subsequent graft tear or injury to the opposite knee. In fact, such injuries are reported at rates of $3 \%$ at 2 years after surgery, and up to $6 \%$ at 5 years. One factor that could contribute to these statistics is anatomy. Some studies have shown that a high posterior tibial slope, or PTS, is a predictor of graft tear or contralateral ACL injury following ACL reconstruction. Others, however, have failed to produce supporting evidence. To explore that relationship, researchers examined more than 2000 ACL reconstructions with patellar tendon autograft, with a mean follow-up of 11.6 years. The PTS was measured prospectively using radiographs by subtracting from 90-degrees the acute angle formed by intersecting lines drawn along the medial tibial plateau and the posterior tibia. Patients experiencing graft tear after primary ACL reconstruction surgery showed statistically significantly higher mean PTS values than patients without subsequent tear. And patients with PTS values of 10-degrees or higher had a statistically significantly higher rate of graft tear than patients with PTS values of 9-degrees or lower. Notably, however, PTS was not found to be a statistically significant predictor of recurrent ACL graft tear rates in patients undergoing revision surgery. In addition, the overall rates of injury to the graft or contralateral knee were low for both primary and revision surgeries. So, while PTS appears to have an effect on primary ACL injury, these results should not be used as support for procedures to correct PTS, such as osteotomy. Osteotomies are invasive and risky and were not evaluated in this study as a protective measure against ACL graft rupture in patients with increased PTS following primary ACL reconstruction. 